\title{
Experimenting the impact of pedestrianisation on urban pollution using tangible agent-based simulations: application to Hoan Kiem district, Hanoi, Vietnam
}

\author{
Arthur Brugière ${ }^{1,2}$, Minh Duc Pham ${ }^{1,2}$, Kevin Chapuis ${ }^{1,3}$, Alexis Drogoul ${ }^{1,2}$, \\ Benoit Gaudou $^{1,2,4}$, Arnaud Grignard ${ }^{5}$, Nicolas Marilleau ${ }^{1}$, and Nguyen-Huu \\ $\operatorname{Tri}^{1,6}$ \\ 1 UMI 209 UMMISCO, Sorbonne Université, IRD, Bondy, France \\ 2 ICTLab, USTH, Vietnam Academy of Science and Technology, Hanoi, Vietnam \\ 3 UMR 228, ESPACE-DEV, IRD, Montpellier, France \\ 4 UMR 5505, IRIT, Université Toulouse 1 Capitole, Toulouse, France \\ 5 City Science, MIT Media Lab, Cambridge, USA \\ ${ }^{6}$ IXXI, ENS Lyon, Lyon, France \\ Contact author: benoit.gaudou@gmail.com
}

\begin{abstract}
The development of permanent or temporary pedestrian areas, whether for leisure or to decrease air pollution, has become an integral part of urban planning in numerous cities around the world. Hanoi, the capital of Vietnam, began to implement its first area, around the iconic Hoan Kiem lake, a few years ago. In most of cases, however, road closure is likely to deport traffic to nearby neighbourhoods with the consequences of intensifying congestion and, possibly, increasing air pollution in these areas. Because this outcome might appear counter-intuitive to most stakeholders, it is becoming more and more necessary to analyse, assess and share the impacts of these developments in terms of traffic and pollution shifts before implementing them. In this project, we used the GAMA platform to build an agent-based model that simulates the traffic, its emissions of air pollutants, and the diffusion of these pollutants in the district of Hoan Kiem. This simulation has been designed so as to serve either as a decision support tool for local authorities or as an awareness-raising tool for the general public: thanks to its display on a physical 3D model of the district, people can effectively and naturally interact with it at public venues. Although more accurate data and more realistic diffusion models are necessary and will need further research in the future, the simulation is already able to reflect traffic and air pollution peaks during rush hours, allowing residents and developers to understand the impact of pedestrianization on air quality in different scenarios.
\end{abstract}

Keywords: Agent-Based Model, Air Pollution, Urban traffic, GAMA Platform, Interactive Simulation 


\section{Introduction}

According to the World Health Organization (WHO), air pollution, particularly in urban areas, is the leading environmental health risk worldwide, causing about one in nine deaths [26]. Awareness of the extent of this phenomenon is recent and still confused, particularly in developing countries where there is an accelerated, and sometimes uncontrolled urban development. Even if urban air pollution sources are multiple (local factories and agriculture burning, or regional atmospheric streams [8]...), the urban traffic and congestion have definitely (even if it is not clearly quantified) an impact on the amount of pollutant in air [39]. Adapted traffic and more generally urban management strategies are thus necessary to reduce traffic-related emissions and inhabitant exposure [5]. The difficulty encountered by urban planners lies not only in the diversity of possible solutions (e.g. development of public transport, infrastructure development, changes in regulations) but also in their acceptance by the inhabitants concerned by the risk and often poorly, or not at all, informed about their effects. Better consultation between planners and residents is therefore a necessary step to avoid finding solutions that are rejected, misunderstood, or even counterproductive.

However, this stage comes up against uncertainty concerning the possible evolutions of the city and the great complexity of the studied phenomena: their understanding, analysis and projections into possible futures are currently a real scientific challenge, particularly because it is difficult to measure and predict the impact of individual and collective adaptation behaviours, whether to pollution or regulations. The complex nature of a city dynamics (in the sense of the complexity science [23]) with the importance of spatial and individual heterogeneities lead us to choose the agent-based modelling [22] approach to tackle this issue. But we argue here that we need to go further to enhance the understanding of the simulated phenomena (both by decision-makers and by random people) and the discussions about result patterns. We chose to make the simulation tangible (by providing a physical substrate) [27] and interactive, so that the user can formulate questions on the simulation it observes and test them in runtime.

Following these principles, we propose in this article a decision-making and consultation support tool based on the design and implementation of an interactive and tangible agent-based simulator. In the frame of the HoanKiemAir project, we couple a 3D printed map of the studied area (the Hoan Kiem district of Hanoi city, Vietnam) used as a screen to show simulations of an agent-based model (implemented using the GAMA platform software [33]) coupling a traffic sub-model and an air pollution sub-model (air pollution is limited to pollutants emitted by vehicles). Users can interact with the simulation using an Android application to modify the traffic and the urban environment and observe, in real time, the impact of these choices on urban air quality. The agent-based model has been designed to be able to take into account individual mobility and adaptation behaviours in order to reproduce Vietnamese urban traffic and its evolution, particularly in the case of new urban planning (e.g. various pedestrian areas implementations). 
The model has previously been presented in details in [28]. We focus this article on the analysis and exploration of the model, with the key objective of providing insights to the question of the impact of alternative pedestrian areas scenarios on the emitted pollution.

The article is organized as follows. Section 2 details the case study: the area of Hoan Kiem district and the problematic linked to the implementation and extension of the pedestrian area around its central lake. Section 3 presents, first, a quick state of the art related to modelling of urban traffic and air pollution, and secondly, an overview of tangible and interactive simulations and in particular the ones used for urban planning. Section 4 presents a summary of the HoanKiemAir agent-based model. The two following sections describe the two possible uses of the model: Section 5 describes its interactive use through the 3D map and the Android application, while Section 6 presents the first results from its sensitivity analysis and exploration. Finally, Section 7 concludes the paper and discusses perspectives and future extensions.

\section{Case study}

For several years, the Hanoi People's Committee drew up a plan to organize some pedestrian zones in Hanoi, where vehicles would be banned on weekends. This plan was tested and implemented in two different areas of the city, one of which is located around the iconic Hoan Kiem Lake, which is the centre of the city old quarter, and the key touristic spot of the city. From 7PM every Friday evening until 2PM on Monday morning, several streets are closed to any vehicle and thus invaded by thousands of pedestrians (see Figures 1a and $1 \mathrm{~b}$ for the map of the closed roads in the current pedestrian area). As time goes by, this area is becoming a popular tourist attraction as well as a gathering point for residents.

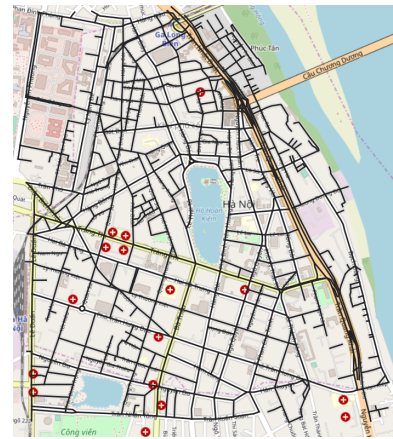

(a) No closed road

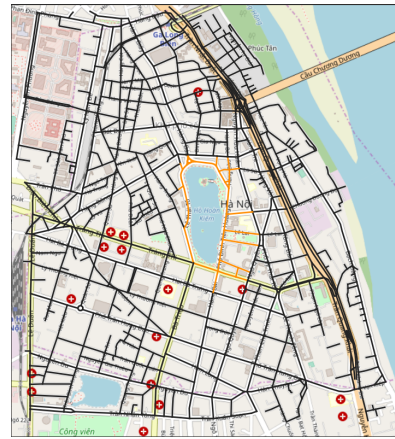

(b) Current area

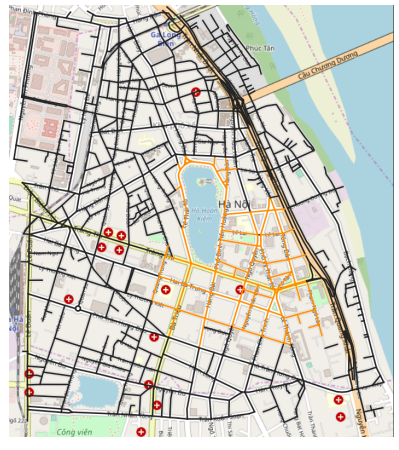

(c) Possible extension plan

Fig. 1: Pedestrian area scenarios: roads in black are opened for vehicles, while those in orange are closed. 
However, in a city as dense as Hanoi, the closure of roads, especially in the city centre, is not without consequences on traffic flows and, albeit indirectly, on the air pollution induced by this temporarily reorganized traffic. While local air quality is clearly improving around the lake and the area have become much more pleasant for pedestrians, the effects of the induced reorganization of the traffic do not have been yet evaluated on the rest of the district and even at the scale of the city. We can suppose that this will lead to a degradation elsewhere, due to the report of the traffic in other streets of the district, but no simulation or visualisation tools are available in order to observe this impact.

Thanks to the popular success of the implementation of the weekend pedestrian area, authorities are thinking about extending the pedestrian area and thus to close more roads during the weekend (one of the possible extension plans is displayed on Figure 1c). Whereas the current plan is focused mainly on the roads around the lake and some small ones close to it, such a new plan could close many streets in the South and the East of the district and have a much more important impact on the traffic.

One of the objective of the HoanKiemAir model is to be able to take into account such alternative closure plans and compute their impact on the traffic and pollution emitted.

\section{Related works}

\subsection{Traffic flow models}

Modelling of traffic (in particular in an urban context) is now a widely spread approach to optimize traffic flow, reduce traffic jams or improve infrastructures, and help urban planning. [16] or [6] identified three main modelling approaches, mainly related to the modelling scale ${ }^{7}$.

Macroscopic models represent traffic flow in the streets as a fluid flow in a pipe. Mathematical models can thus be used to describe the evolution of some macroscopic values of the flow: density, average speed, volume... The paradigmatic model is LWR $[29,21]$. Although they can produce realistic outputs with low computational cost and in a deterministic way, there are not expressive enough to represent heterogeneities in terms of vehicles and drivers' behaviours.

On the other hand, microscopic models describe vehicles individually, with their individual attributes (location, speed, acceleration...) and behaviours. As an example the classical NaSch model [24] has first been implemented as a Cellular Automaton and later as an agent-based model [3]: each car is represented individually with its own speed. Its behaviour is to go straight, accelerating as much as it can (given an acceleration parameter) and decreasing its speed when another vehicle is before it. Such models are highly expressive, able to represent any kind of vehicles, drivers' behaviours and traffic situations. However, computation load can be very high as the number of vehicles in the network becomes larger.

\footnotetext{
7 [37] illustrates a genealogy of traffic flow models.
} 
Finally, mesoscopic models are hybrid models, combining features of the two previous approaches. A hybrid model can for example model vehicles not as individuals but as small groups. They allow vehicles to make certain decisions, but at the same time limit the level of detail on their behaviours. An example of mesoscopic model is the classical Underwood model [35] which considers individual vehicles, but their speed is constrained by the total number of vehicles on a road edge. Another interesting approach relies on the coupling between several models from different approaches in order to build a model that can adapt the simulated depending on the traffic and on the situation [4].

In the HoanKiemAir, we chose the mesoscopic approach by developing an agent-based model (ABM) [22], i.e. a model representing the studied system at an individual level: each vehicle is represented, with its individuals features and behaviours (perception, decision-making process, learning...) ${ }^{8}$. The overall dynamics is expected to emerge at a macroscopic level from individual agents' behaviours and interactions. ABMs have the advantage to be easily spatialized (in a continuous 2D or 3D environment, or on a discrete grid of graph). ABMs have now a wide variety of applications, from physical and life sciences to economics and social sciences. Such agent-based models has been developed for example for evacuation using vehicles [7] or to investigate various mobility means [12]. Other advantages of ABMs are their flexibility and expressivity, e.g. to easily integrate urban management policies or to be coupled with various other modelling approaches, such as the ones used to model air pollution.

\subsection{Pollution model}

To model air pollution, two main dynamics have to be taken into account: emission and dispersion. An emission model calculates the amount of pollutants released by any source (factories, vehicles...), whereas dispersion models aim to describe how air pollutants disperse in the atmosphere.

When considering air pollution in an urban context, a widely used dispersion model is the Operational Street Pollution Model (OSPM) [15], suitable for areas with street canyons (i.e. streets surrounded by tall buildings). It assumes that two factors contribute to the concentration of pollutant in a street canyon: traffic flow (with direct emission from vehicles and air recirculation in the street canyon) and other sources (pollution from other streets, factories, power plants...). The European emission model COPERT IV [25] is integrated into OSPM as an emission module. This model (mainly funded by the European Union) is now recognized as a standard in both the academic and industrial communities. The emission values are computed based on fuel type, emission factor as well as vehicle type and diurnal traffic frequency. An example of integration of such a model with an agent-based traffic model can be found in the MarrakAir project [10] aiming at investigating the impact of the number, type and age of vehicles on air pollution in the case-study of Marrakesh (Morocco).

\footnotetext{
8 The model is mesoscopic because we rely on the Underwood model to compute vehicle speeds.
} 
Whereas OSPM is mainly focused on European context, [34] applied it on the Vietnamese context (in particular on the case study of Hanoi) and provided data about Vietnamese vehicles emissions. Using a simplified version of OSPM and data from the previous study, [30] have proposed a GAMA simulation to study traffic-induced air pollution in Hanoi, Vietnam.

\subsection{Tangible User Interface and simulation}

The idea of tangible and interactive simulations is to use a physical support (often named Tangible User Interface (TUI), see [11] for an attempt of classifications) such as a 3D printed map, sandbox, laser cut map or any other physical objects [31] to display digital information coming from a simulation. The benefits of such tangible objects to help the user to think spatial information and dynamics has now been shown (see Chapter 1 of [27] for more details about the various cognitive benefits). Similar benefits have been demonstrated in an educational context [38].

TUI can take many shapes [11] and often the setup is specific to the case study. When considering simulations supported by a TUI, a few main components can be identified in most of the projects:

(i) the simulation, representing the virtual world and its (spatial) evolution; it should be able to manage inputs from the user, modify the virtual world in accordance and take into account these modifications in future computation.

(ii) controllers, that are devices controlled by the user to modify the simulations or sensors perceiving some modifications made in the environment by the user; examples include 3D Kinect camera (to detect modifications in a physical environment, such as a sandbox) [31,38], physical objects with RFID tags [17,20], Lego blocks $[2,13]$ or application on tablet $[10,19]$.

(iii) physical objects, that can be a simple 3D map of the virtual environment used as a screen to display the simulation $[2,10,13,27]^{9}$, a fully modifiable environment (as a Sandbox [38], that works also as a controller, through the camera), or a set of physical objects representing elements in the simulation and that the user can manipulate (e.g. traffic lights in [20] or buildings in [31]);

(iv) visualisation supports, in addition to a display of the simulation on the 3 D map $[2,10,13,27]$ or Sandbox [38], or on an interactive table (such as the TangiSense table [18]), it is common to add screens to display additional information (3D rending of the environment in Tangible Landscape [27] or additional indicators [17]).

Such an approach is now used in most of the topics that rely on a spatial representation of the case studies and that can be tackled by simulation.

\footnotetext{
${ }^{9}[19]$ shown that displaying a flood simulation even on a flat table for participative simulations provides a much better immersion for participants and increase discussions than displaying it on a wall.
} 
One of the first implementation of such an approach is perhaps the Augmented Reality sand boxes, such as the one developed in the Lake visualisation $3 \mathrm{D}$ project ${ }^{10}$. Given a sandbox and a Kinect camera (a low-cost camera able to detect movements in $3 \mathrm{D}$ ), users can play with the sand to modify the ground topology, by digging rivers or "building" mountains or volcanoes. The camera is able to identify in real-time modifications of the elevation and given its values, fill the gaps with water. [38] built such a setup and evaluate its positive impact in geology classes on students to make them understand some key concepts and interactions between elements (e.g. water with the landscape). Many other examples of Tangible User Interface in landscape study are presented in [27].

Concerning urban case studies, one of the first work we can identify [31] was dedicated to simulate wind streams in a city, depending on the buildings' location. It is composed on a 3D (laser cut map) map of the city, a Kinect camera to identify the position of buildings and a fluid simulator, taking as input these buildings and computing the wind flow. [20] used an interactive table (TangiSense table [18]) to simulate simple traffic situations; interactions with the simulation is made through some physical objects (traffic lights...) with an RFID tag. Later the CityScience research group of the MIT Media Lab developed several projects $[2,13]$ to give the possibility to visualize the link between urban planning and organization, mobility modes and traffic. [10] focuses on the link between traffic (in particular related to vehicle numbers and states) and air pollution.

Finally, [17] use a tangible table with RFID tag-based physical objects to define collaborative simulations to help crisis management.

\section{Model}

We present in this section the key elements of the HoanKiemAir model ${ }^{11}$, developed using the agent-based modelling and simulation GAMA platform [33]. An extensive description of the model is presented in [28] using the standard O.D.D. (Overview, Design concepts, Details) protocol [14].

\subsection{Overview of the model}

The model aims to simulate the interrelation between traffic, air pollution, and road management (in terms of pedestrian areas scenarios) in the Hoan Kiem district (Hanoi). The main question tackled by the model is: how the various pedestrian area scenarios and the evolution of the number of vehicles influence congestion and air pollution in the area?

${ }^{10}$ LakeViz3D project: http://lakeviz.org

11 The model is open-source and available for its GitHub repository: https:/github. com/WARMTeam/HoanKiemAir. 


\subsection{Description of the model entities}

The model is composed of two main dynamics, the traffic sub-model and the pollution sub-model, influenced by pedestrianization scenarios.

The traffic sub-model generates the movement of vehicles on a road network. It is composed of two types of agents: vehicle agents move on road edges toward an intersection target.

The pollution sub-model is divided into an emission model and a dispersion model. The emission model is managed by a single agent pollutant_manager, which takes the output of the traffic model, calculates the amount of pollutants emitted by vehicles, and then passes them to the dispersion model. The dispersion model is based on a collection of agents pollutant_cell, which represent airborne packages. Each pollutant_cell has a volume (depending on its area) in order to compute a pollution concentration in each one. We consider here two kinds of dispersion models (that will be evaluated in the next section):

(i) pollutant_cell agents are cells of a grid covering all the space. Each cell will thus get the pollution from the road edges it overlaps and diffuses to its 8 neighbour cells.

(iii) pollutant_cell agents are buffers on top of each road edges. Each cell will thus get the pollution from the associated road, and will diffuse to the neighbour pollutant_cell. This model makes the assumption that pollution that pollutants cannot be dispersed over building rooftops, but only through streets.

\subsection{Description of the model processes}

In a simulation cycle, the agents of the traffic model are scheduled first, followed by those of the emission and dispersion model (Figure 2 illustrates the various steps of a simulation cycle). One simulation step represents by default 16 seconds, in order to be able to observe vehicles moving on the roads. When we want to observe the influence of the number of vehicles over time in the day on the pollution, we need to simulate on a period of several days. In order to keep the simulations interesting for users we choose in this case a simulation step representing 5 minutes.

In the traffic model, the road agents are executed first, updating their congestion levels. We choose indeed to use a traffic flow model close to the Underwood model [35]: roads compute a congestion factor, which will influence the speed of vehicles moving on it. Finally, the vehicle agents move to their target (a vertex of the road network, i.e. a road intersection) on the traffic graph. The vehicles move using built-in features implemented in the GAMA platform. When a vehicle reaches its target, it will choose a new intersection as next target.

Once all vehicle agents have completed their movement, the pollutant_manager agent computes the amount of traffic-induced pollutants and stores them in pollutant_cell agents. Then, it decreases the 


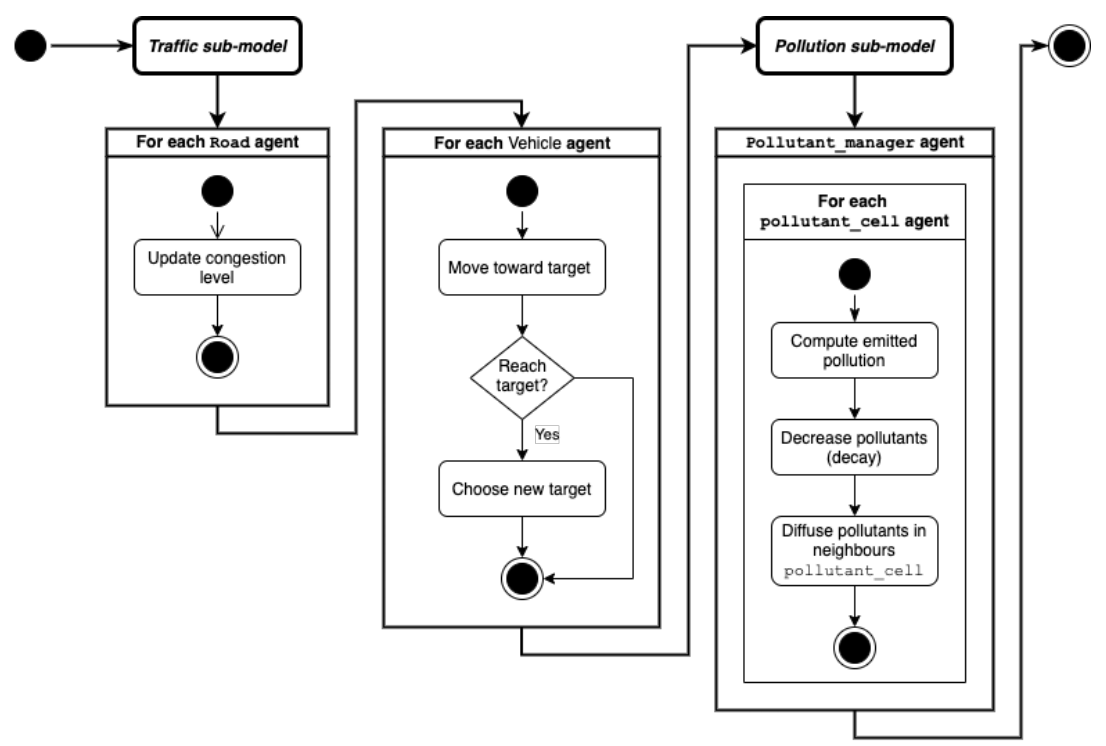

Fig. 2: Flowchart of the execution of one cycle of the HoanKiemAir model.

pollutant values in these cells according to a predefined decay rate $^{12}$ and disperses the pollutants in the neighbouring cells (the dispersion algorithm depends on the model implementation).

\subsection{Input data}

The traffic and pollution sub-models are executed only on the Hoan Kiem district (an area of $2506 \mathrm{~m} \times 2779 \mathrm{~m}$ ). Nevertheless, to provide some landmarks for the users, we also display spatial elements on a wider area (a rectangle of $5458 \mathrm{~m} \times$ $3052 \mathrm{~m}$ ) that will not have any influence on the simulation. Figure 7 shows all the spatial data used: shapefiles impacting the simulation (roads and buildings) and the additional shapefiles loaded for contextual and visualisation purpose (lakes, rivers, special buildings and main roads shapefiles). These additional roads and the roads in the Hoan Kiem district are stored in 2 different shapefiles in order that only roads in the considered area are used as support of the traffic model.

Most of the spatial data (except buildings that have been manually sketched from satellite images) come from OpenStreetMap (OSM) ${ }^{13}$ data source. In addition to geometric information, the OSM data also contain various attributes. For example, as far as roads are concerned, OSM can provide the number of lanes,

\footnotetext{
12 To be more precise, the decay factor has to be understood as the remaining ratio of pollutant after decay. If it values 0.99 , this means that after decay, $99 \%$ of pollutants are kept or in other words, that they have been reduced by $1 \%$.

13 OpenStreetMap: www.openstreetmap.org.
} 
maximum allowed speed, one-way or two-way, traffic light availability, etc. Although OSM provides some information for some roads, most of the values are missing, so we have defined a set of default values: the maximum permitted speed of $60 \mathrm{~km} / \mathrm{h} . .$.

Finally, the hourly distribution data of traffic (see Figure 3 ) is provided by [1]. They estimated the number of trips per hour throughout the day and give us a realistic traffic flow over a full day.

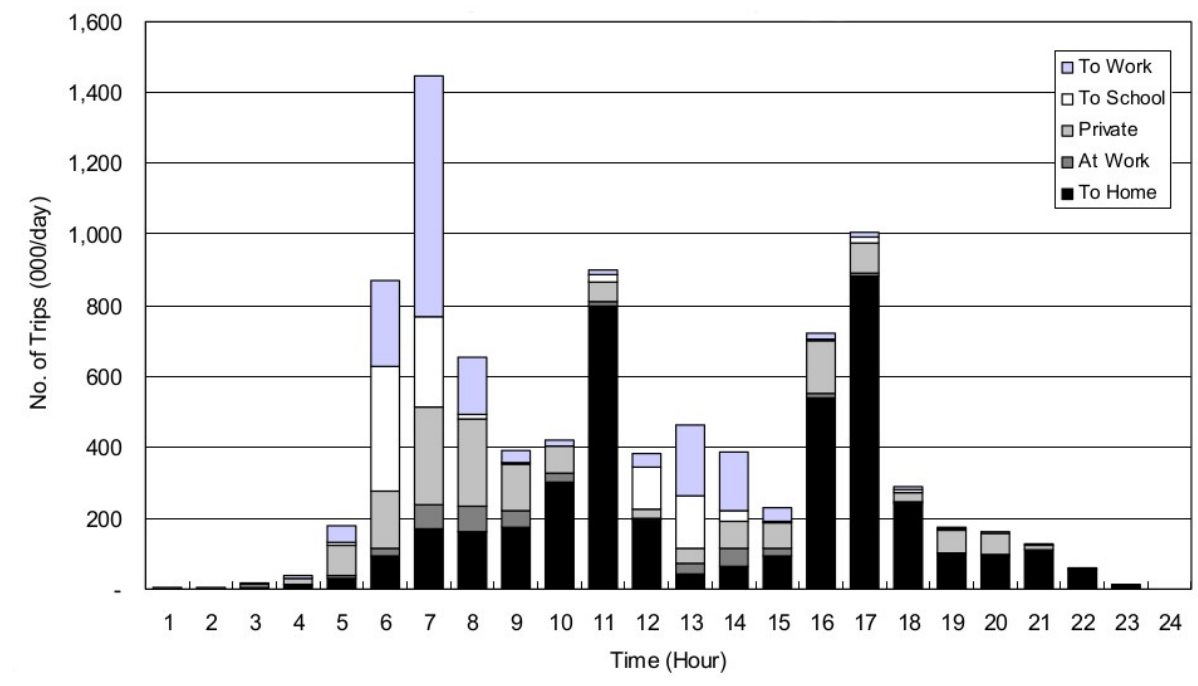

Fig. 3: Example of hourly traffic distribution (from [1]).

\subsection{Outputs}

To make pollutant values easy to interpret, an Air Quality Index (AQI) value is calculated from the amount of each pollutant. We use the method given by [36]:

$$
A Q I^{h}=\max _{x}\left(A Q I_{x}^{h}\right) \text { with } A Q I_{x}^{h}=\frac{T S_{x}^{h}}{Q C_{x}} * 100
$$

where $A Q I_{x}^{h}$ is the AQI of a pollutant $x$ at hour $h, T S_{x}^{h}$ is the measured average amount of pollutant $x$ in $h$, and $Q C_{x}$ is the allowed average amount of pollutant $x$ in an hour (Table 1). Ranges of AQI are associated to colours, as illustrated in Figure 4b. The pollutant_cell agents are not made visible and the pollution is displayed on buildings (see Figure 4a for an example on the 3D map).

In addition, in order to observe the overall evolution over time of the AQI, we display a chart of the maximum value of AQI in the district over time. Figure 6 shows the full simulation display. 
Table 1: Allowed hourly average amount for different pollutants (measured in $\left.\mu g / \mathrm{m}^{3}\right)[36]$

$$
\begin{array}{llll}
\hline \mathbf{C O} & \mathbf{N O}_{\mathbf{x}} & \mathbf{S O}_{\mathbf{2}} & \mathbf{P M 1 0} \\
\hline 30000 & 200 & 350 & 300 \\
\hline
\end{array}
$$

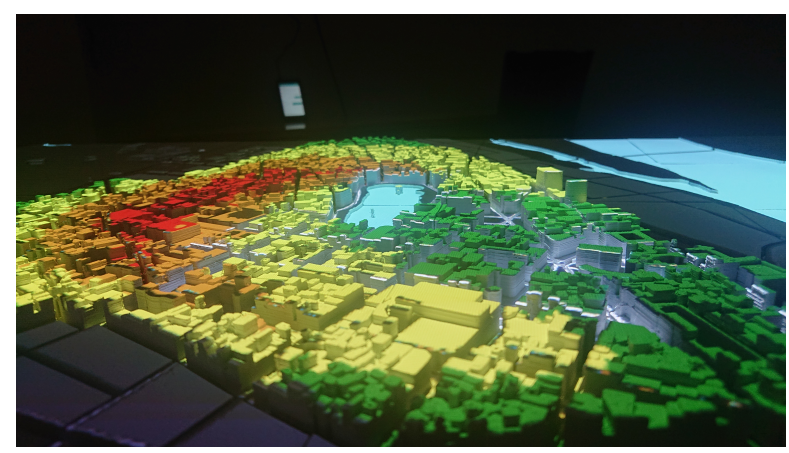

(a) 3D map

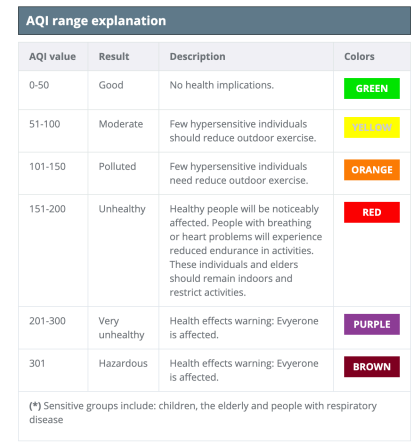

(b) AQI color scale

Fig. 4: (a) Example of display on the 3D map (using colors defined in the AQI scale (b). Source: https://moitruongthudo.vn/).

\section{$5 \quad$ Interactive simulations}

Inspired by the MarrakAir project [10], we built a full setup for tangible and interactive simulations that is accessible by any random user. It consists of three main components:

- The simulation of traffic and pollution implemented on GAMA platform;

- The tangible part is managed by a 3D-printed map of a part of Hanoi city, with a focus on the Hoan Kiem district, which is used as visualisation support of the simulation;

- The controller is an Android application, installed on a tablet, that communicates with the simulation via networking, allowing users to modify in runtime simulation parameters and the visualisation mode.

Figure 5 illustrates the whole setup when it was located at the French Embassy in Hanoi during the summer 2019. It shows in particular the video projector located on top of the 3D map.

\section{$5.13 \mathrm{D}$ models}

The simulation is running only on the Hoan Kiem district (cf. Figure 1), nevertheless, as the 3D map is the only place to display information for the users, we made the choice to build the 3D map on an extended area. This brings two main 


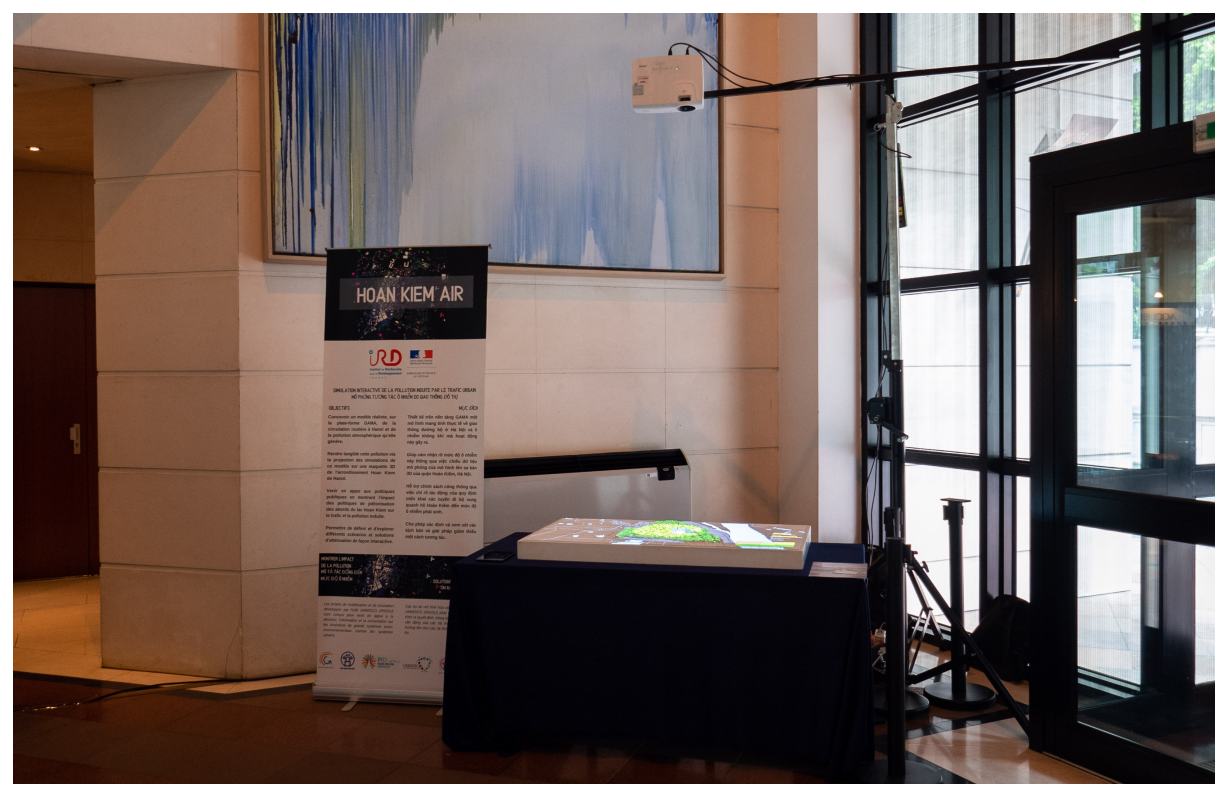

Fig. 5: The full setup for tangible and interactive simulations (including the 3D map, the tablet with the Android application, the projector).

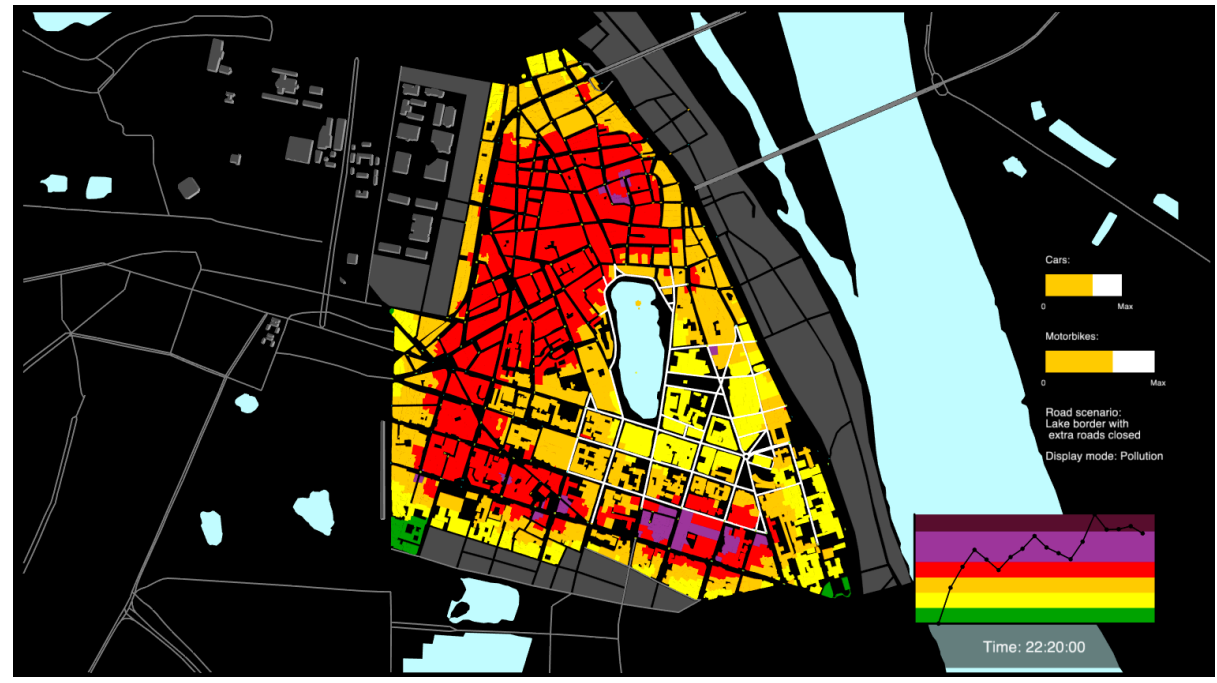

Fig. 6: Screenshot of the full simulation, including the pollution map, the pollution charts and the value of the pedestrian scenario, number of cars and of motorbikes parameters. 
advantages: (i) it allows us to display contextual elements (the Red River on the right, and some of the most well-known buildings of Hanoi on the left, some main roads and bridges of Hanoi...) in order to help users to locate in space; (ii) it provides empty space to provide more information, such as the state of parameters (number of vehicles, the kind of scenario...) and plots (in particular related to the evolution of the pollution). Figure 6 illustrates this combination of parameter values, (air pollution) plot and the display of the agents ${ }^{14}$.

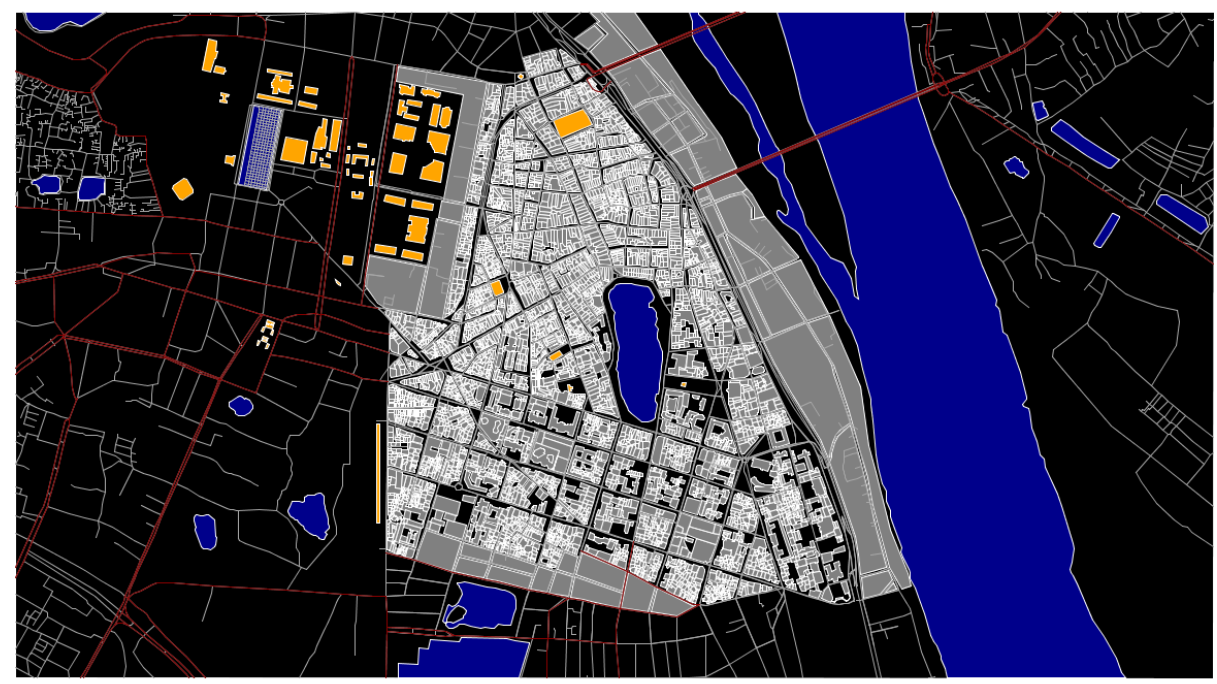

Fig. 7: Data used to build the 3D map: blue elements are rivers and lakes, grey elements are buildings, orange elements are special buildings (markets, Literature Temple, Ho Chi Minh Mausoleum, the National Assembly building, Presidential palace...), white lines represent roads (only the roads inside the Hoan Kiem District are used for traffic simulations), red lines are the main roads (roads outside Hoan Kiem district that are displayed in the simulation).

To produce the 3D models that have been printed, we use some shapefiles used in the simulation (cf. Figure 7 for an exhaustive description of the dataset used to produce the $3 \mathrm{D}$ map). The main challenge was to transform this set of

${ }^{14}$ A video is available to illustrate the dynamics of simulations at the address: https://youtu.be/U2w0GtLHACU. 
shapefiles into a set of tiles in the STL format ${ }^{15}$. Here are the steps to create them $^{16}$ :

1. Prepare the shapefile. First, all the shapefiles are merged in a single one (roads, buildings, rivers and lakes). The roads, which are represented as a polylines are buffered in order to give them a surface. The empty holes are then filled with polygons to represent the base of the 3D map, where there are no buildings, roads or lakes. All polygons are assigned a height value that follow this ordering: (road, lake, river) $<$ base $<$ building. We choose: $0.3 \mathrm{~cm}$ for roads, lakes and rives, $0.5 \mathrm{~cm}$ for the base, and $0.8 \mathrm{~cm}$ for special buildings. For the buildings, we do not have data about their elevation, but when looking at the Hoan Kiem district, it appears that buildings are highly heterogeneous in the area. We thus choose to give a random elevation value between $1 \mathrm{~cm}$ and $1.6 \mathrm{~cm}$ to reproduce this heterogeneity. Finally, we give the size of $2.5 \mathrm{~cm}$ to some specific buildings (the higher buildings in the area), in order to help users to recognize some key points on the map. It was important to keep a low elevation for the elements of the 3D map, to prevent from having huge shadows when the simulation will be displayed on the $3 \mathrm{D}$ map.

2. Generate a single STL file. From the shapefile produced in the previous step, we produce a 3D model file (STL format) with dimensions of approximately $122 \mathrm{~cm}$ by $64 \mathrm{~cm}$.

3. Split the STL file in tiles. As the 3D map is too big to be printed in one go by standard 3D printers, the STL file generated in the previous step is split in square tiles of $16 \mathrm{~cm}$ per $16 \mathrm{~cm}$ following a $4 \times 7$ grid as presented in Figure 8.

\subsection{Remote control through an Android application}

As we expect random users to interact in an easy and intuitive way with the simulations, we choose not to let them manipulate directly the simulation interface and have developed an easy-to-use remote control application (running on an Android tablet). In order to keep the application intuitive, we limited the number of parameters accessible by users to the minimum ones: (i) the number of vehicles (motorbikes and cars), (ii) the pedestrian area scenarios and (iii) the visualisation mode (traffic or air pollution).

For communication between the application and GAMA, the Message Queuing Telemetry Transport (MQTT) protocol is used. It is a fast and lightweight publish / subscribe messaging protocol, commonly used in IoT projects. Basically, clients exchange messages through a server (often called a broker). A client

${ }^{15} \mathrm{STL}$ is a datafile format describing $3 \mathrm{D}$ objects and very common as an input for $3 \mathrm{D}$ printers.

16 The full process and in particular the various softwares used are documented on the HoanKiemAir GitHub repository: https:/github.com/WARMTeam/HoanKiemAir/ tree/master/stl_convert 


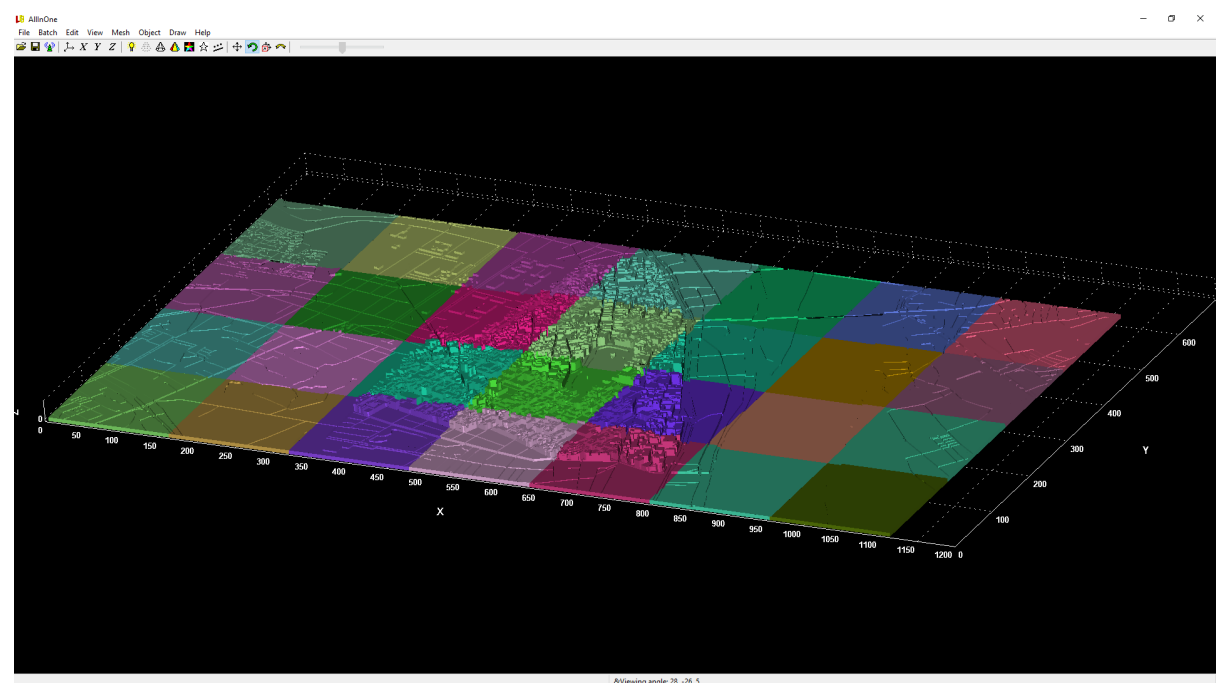

Fig. 8: Grid cut of the 3D map.

can publish a message with a topic to the broker, then the broker will distribute that message to clients that are subscribed to the topic. In our setup, an Apache ActiveMQ ${ }^{17}$ server is executed on the same computer as the GAMA simulator. Both the Android application and the GAMA simulator act as MQTT clients. An external plugin is provided by the GAMA community and implements communication through the MQTT protocol: it allows any agent to become an MQTT client, and thus to connect to an MQTT broker, publish messages and subscribe to certain topics.

Once the application is connected to the broker, it will publish messages with pre-defined topics when users modify any parameter value. A dedicated agent in the simulation receives these topics and modify the corresponding parameters (this can also induce more changes in the simulation, such as the creation or destruction of some agents).

\section{Experiments}

The HoanKiemAir simulator has been designed as an awareness-rising and comprehension-support system. As a consequence, simulations should be both scientifically grounded but also able to deliver its key messages to any users in an interactive way (and thus in a limited among of time). It should thus be explored to identify the parameter ranges fulfilling these two objectives. Finally, this exploration should be designed keeping in mind that the ultimate objective

17 Apache ActiveMQ is an implementation of an MQTT broker, see http://activemq. apache.org/ 
is to be able to provide insights about the impact of the pedestrian area scenarios on the air pollution quantity and distribution.

Given the high number of parameters (see next section for a detailed overview), we were not able to run a global sensitivity analysis. We thus performed some local analyses around the parameter values chosen empirically for the various exhibitions.

As the model is stochastic (mainly due to moving vehicles), we first investigate its sensibility to this randomness factor (Section 6.2). In particular, we need to check that simulations outputs are not qualitatively or quantitatively significantly different among replications. Finally, we will determine the number of replications needed to be launched in the following experiments. As stated in Section 4.3, several simulation step duration can be used in the different execution modes. In Section 6.3, we evaluate the influence of the step duration to check that the simulations keep close behaviours with different values.

Section 6.4 then investigates effects of pollution models and their parameters (decay and diffusion rates). Section 6.5 explores the impact of the number of vehicles on AQI indicators in order to identify whether there is some qualitative changes when the number of vehicles increase and if the AQI have an upper bound. The Section 6.6 then explores the impact of the evolution of the number of vehicles during the simulation (using the daytime traffic scenario). In particular, we aim at identifying whether there is a bootstrap period.

Finally, Section 6.7 explores the impact of the pedestrian area scenario by comparing the three scenarios, but also how the model behaves when the scenario changes during the simulation (to study what happens when the user switches between scenarios, or when we simulate a week the switch during the week-end).

All the experiments have been executed in headless mode on the following hardware configurations:

- Laptop MacBook Pro 2017

- CPU - Intel(R) Core(TM) i7-7820HQ CPU @ 2.90GHz (8 virtual cores)

- RAM - 16 Go

- Operating System - MacOS 10.15.5 Catalina

- Laptop ASUSTeK GL553VE

- CPU - Intel(R) Core(TM) i7-7700HQ CPU @ 2.80GHz (8 virtual cores)

- RAM - 16 Go

- Operating System - Linux Ubuntu

- Two nodes on universitary HPC

- CPU - Intel(R) Xeon(R) CPU E5-2620 v4 @ 2.10GHz (16 virtual cores)

- RAM - 24 Go

- Operating System - Linux Debian

\subsection{Parameters and indicators}

Parameters. The model has been designed to be exhibited during an undetermined time. As a consequence, a simulation stop condition cannot be defined as a specific situation; we thus define it as a final simulation step at which 
outputs are (expected to be) stable. In addition, given the model stochasticity, several replications (i.e. simulations launched with same parameter values) have to be launched to get significant results: this number of replications has thus to be determined before going further in the exploration. Finally, we explore the impact of the simulation step duration, in order to check that it can be adapted.

The evolution of the number of vehicles in the area is controlled by the parameter traffic scenario, that can take 2 values: constant traffic ${ }^{18}$ or daytime traffic. The default value is set to constant traffic, which means that the number of vehicles is constant and determined by the three parameters: number of cars (by default equal to 700), number of motorbikes (by default equal to 2000), and the traffic factor (by default equal to 1) which multiplies the initial number of vehicle (car and motorbike) by a given factor. This factor will vary to explore the impact of the number of vehicles. When daytime traffic is selected, the number of vehicles evolves with the hour in the day (following data presented in Figure 3).

As far as pollution diffusion is concerned, we will explore the two models implemented (depending on the pollution diffusion model parameter): grid and roads. By default, the model is grid. The two parameters influencing these pollution models are the decay rate (a real value between 0 and 1.0, with the default value set at 0.99 ) and the diffusion rate parameter (a real value between 0.0 and 0.12 , to keep the uniform diffusion conservative, and set to 0.05 by default).

The set of closed roads is defined by the pedestrian area scenario. It can take 3 values: no pedestrian area (default value), current scenario, extended area.

Indicators. As it is the key quantity impacted by all the dynamics of the model, all the indicators are related to the air pollution, represented by the AQI value (cf. Section 4.5). First implemented indicators are thus the mean and standard deviation of AQI over all the buildings (as the AQI is computed on buildings in the simulation). Another interesting indicator would be the total amount of pollution (to assess whether closing roads increase total pollution or only move it). Nevertheless, as the number of buildings is constant, the AQI average and total values have the same evolution, and we only compute the former one. Finally, to observe the distribution of pollution values, we also compute the maximum and minimum value of AQI. As these values continue to change, even when the dynamics is stabilized, we compute the average value over last steps.

For each parameters values, we will run several replications. For each of them, we will save the value of each indicator at each simulation step. We will then plot, for each indicator and parameters configuration, the evolution over the simulation of the average value (over all the replications) as a line. It will

$\overline{18}$ constant traffic is the mode also used when we want the user to change itself the number of vehicles through the application. 
be surrounded by an extreme values interval bounded by the maximum and minimum value of the indicator among all the replications.

\subsection{Stochasticity sensitivity analysis}

This experiment aims at exploring the impact of the stochasticity on the simulation outputs. The objective is twofold. First we want to identify some meta parameters that will be used in the following experiments: the number of steps that need to be executed before reaching a quite stable state (when the number of vehicles is constant) and the number of replications that will be necessary in the following experiments in order to find results that are statistically meaningful. Second, we want to determine the stability of the simulation results. In particular, if the results show a huge instability, it will be difficult for simulation users to understand what they observe.

First, to find the maximum step for the simulations, we run 4 replications (with a final step of 10000) and observe the average values of the mean and standard deviation of AQI over the 4 replications (cf. Figure 9). We can observe that the values change a lot between steps 0 and 500. As the simulation is initially empty of pollutants and of vehicles, when the first vehicles emit pollution, the AQI increases quickly. After these first steps, the simulation reaches an equilibrium between pollutant emission (that tends to increase the AQI) and the pollution diffusion and decay (that tend to decrease the AQI) dynamics. After step 1000, we observe a continuous decrease of the mean AQI value, toward a stable value (around step 9000). Nevertheless, from step 1000 and 10000, we cannot observe any qualitative change in the simulation outputs. In a trade-off between the computation capabilities and the parameter space to explore, we will thus consider in most of the following experimentation a final step at 1500 .

Even if we do not aim at quantitatively exploring or calibrating the model in this paper, we can notice that the effect of stochasticity increases with the number of steps (the extreme values interval amplitude increase with the number of steps).

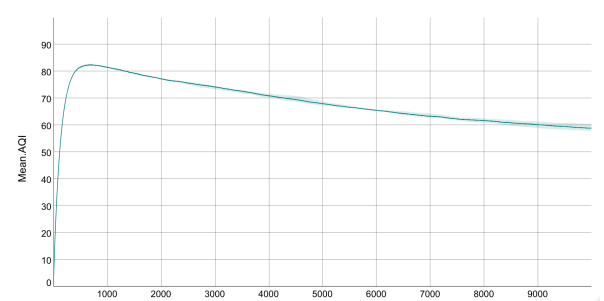

(a) Mean of AQI values.

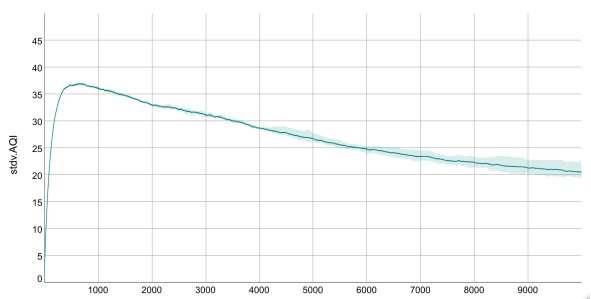

(b) Standard deviation of AQI values.

Fig. 9: Mean and standard deviation values of the AQI in buildings over 4 replications of 10000-step simulations (with a extreme values interval limited by the minimum and maximum values). 
In order to investigate the number of needed replications, we adapt the method detailed in [9] using the standard error of the mean. The standard error of the mean $\sigma_{\bar{x}}$ is defined on a given indicator (e.g. mean, standard deviation of AQI) as:

$$
\sigma_{\bar{x}}=\frac{\sigma}{\sqrt{N}}
$$

where $\sigma$ is the standard deviation of a given indicator and $N$ the number of replications considered.

The main idea is to compute this standard error for an increasing number of replications and to find a threshold number where adding more replications does not bring more information nor a better stability.

So we first compute a big number of replications (here we ran 100 replications as a first attempt). Then incrementally we compute the standard error on an increasing number $N$ of replications from 2 to 100 . For each $N$, in order to prevent biases in the chosen replications, we compute the average of the standard errors over 10 picks of $N$ replications. The results for the 4 indicators (mean, standard deviation, maximum and minimum of AQI) are displayed in Figure 10. We can observe that on all the indicators, the main standard error's evolution are before 10 replications. After 20 replications, the standard errors seem to begin to stabilize and 60 replications seem to be the threshold after which adding more replications do not bring any new information. As a consequence, depending on the exploration purpose and on the required precision, one of these 3 thresholds could be chosen.
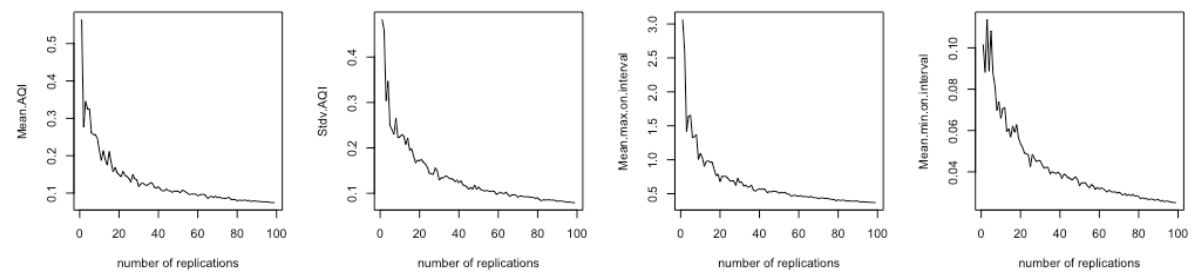

Fig. 10: Standard errors on mean, standard deviation, max, and min AQI, over 100 replications. Figures show a very fast decrease before 10 replications, a beginning of stabilization around 20 replications. After 60 replications, standard errors are stable and additional replications do not bring more information.

Again, following our objective of qualitative exploration and in order to be able to explore a bigger parameter space, we will limit to 10 replications for each parameter value. Further exploration could choose 20 or 60 replications for precision's sake. 


\subsection{Impact of the step duration}

This second experiment aims at exploring the impact of the simulation step duration over the results, but we went to 5 minutes to simulate the daytime traffic.

The objective of this experiment is to evaluate the impact of the simulation step duration on the air pollution index evolution: we aim at checking that the model behavior is still acceptable when changing the simulation step. To this purpose, we consider a single parameter (the step duration) and run 10 replications for each value of the step duration among $\{16 s, 30 s, 60 s, 120 s, 180 s, 300 s\}$. We plot the various indicators (mean, standard deviation and min and max of AQI) for each step duration (Figures 11).

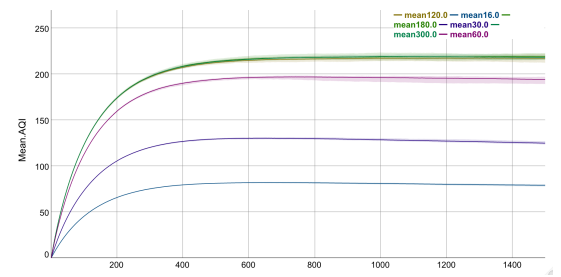

(a) Mean of AQI

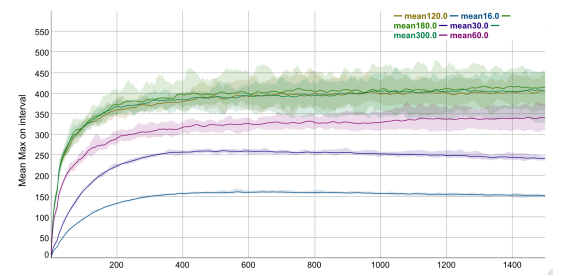

(c) Max of AQI

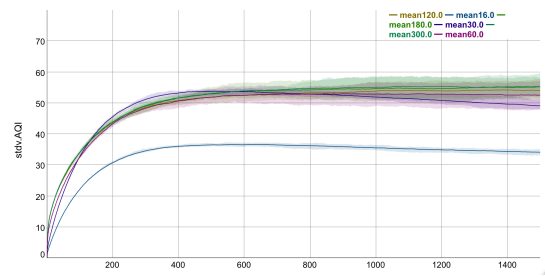

(b) Standard Deviation of AQI

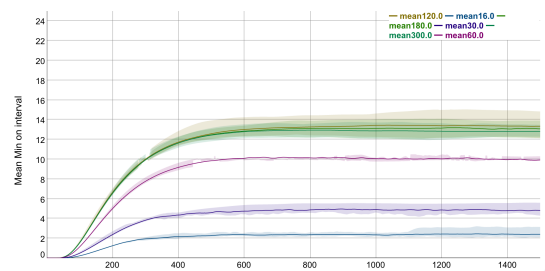

(d) Min of AQI

Fig. 11: Mean values of the mean, standard deviation, and min and max of AQI indicators for the step duration in $\{16 s, 30 s, 60 s, 120 s, 180 s, 300 s\}$. Figures illustrate the impact of the simulation step on results: when the step duration becomes longer, all indicators increase. The values converge when the step duration is greater then 120 s.

We can observe that the simulation step has an impact on the AQI values: the values for all the indicators increase with the simulation step duration. As in the previous experiments, the stochasticity has more impact on results when the simulation step is longer: this can be observed by the extreme values interval becoming bigger with the step duration. We can also observe a convergence of the indicators values: above 120 s, the step duration does not impact results anymore (and even above 60s for standard deviation). Finally, and this is the most important result of this experiment, even if the indicators values are altered by the simulation step duration, the qualitative evolution of the AQI is the same 
whatever the step duration value is. To sum up, this experiment shows that, in the following experiments, we can modify this duration step without changing the behavior of the simulation. Nevertheless, we need to keep in mind that the AQI values will be altered.

\subsection{Effect of the pollution diffusion model}

As presented previously, two diffusion models have been implemented, each of them depending on 2 parameters: decay and diffusion rates. This experiment aims at evaluating the qualitative impact of these parameters on the simulation outputs. We thus explore the evolution of AQI indicators for all the combinations of diffusion model (among $\{$ grid, roads $\}$ ), decay rate (among $\{0.01,0.1,0.3,0.5,0.7,0.9,0.99\})$ and diffusion rate (among $\{0.02,0.04,0.06,0.08,0.1,0.12\})$. For each combination of parameter values, 10 replications are launched. Results are summarized in Figure 12 for the grid model and Figure 13 for the roads model.

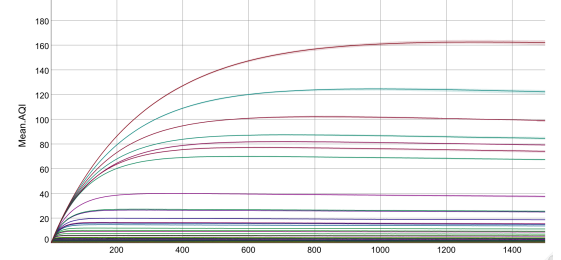

(a) Mean of AQI

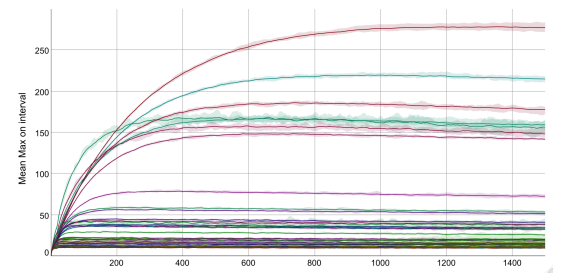

(c) Max of AQI

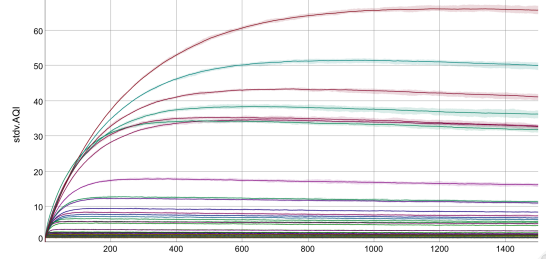

(b) Standard Deviation of AQI

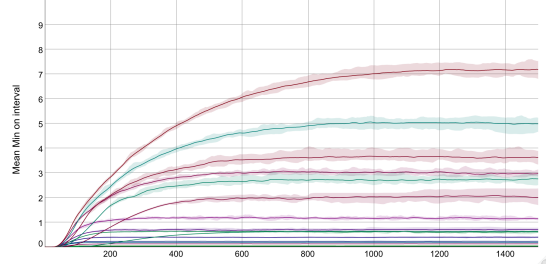

(d) Min of AQI

Fig. 12: Mean values of AQI indicators for the grid diffusion model with decay rate among $\{0.01,0.1,0.3,0.5,0.7,0.9,0.99\}$ and diffusion rate among $\{0.02,0.04,0.06,0.08,0.1,0.12\}$. Some groups of time series appear (with the same decay value) appear for standard deviation and max of AQI in particular, giving the insight that the model is more sensitive to this parameter.

Grid diffusion model. The Figures 12 display the 42 time series produced by this experiment. First no quantitative change appears when exploring all the parameter values. Second, "groups" of series can be observed (in particular of 
the standard deviation and maximum), which could come from the fact that one of the 2 parameters has more influence than the other one.

To confirm this observation, and thus assess the impact of each parameter on the results, we conduct an ANalysis Of the VAriance (ANOVA) [32] at step $=500$. To check the impact of this step value choice, we have computed the ANOVA for other step values and get very close results. Table 2 summarizes the relative impact of each factor (parameters Decay and Diffusion, the randomness (Residuals)) and combination of factors on the dispersion of values. The analysis shows clearly that the impact of the Diffusion parameter and the combination of the parameters Decay-Diffusion have a limited influence on all the indicators. They are mainly impacted by the Decay parameter and the randomness (which has even a more important effect). This means that it is really important to be careful in the choice of the Decay parameter as it will highly impact the values of the AQI.

\begin{tabular}{|c|c|c|c|c|}
\hline & mean & stdDev & max & min \\
\hline \hline Decay & 41.16 & 43.37 & 46.00 & 30.41 \\
\hline Diffusion & 9.04 & 7.40 & 5.89 & 11.91 \\
\hline Decay:Diffusion & 3.91 & 2.74 & 1.64 & 12.17 \\
\hline Residuals & 45.89 & 46.50 & 46.47 & 45.51 \\
\hline
\end{tabular}

Table 2: Results of the ANOVA with the grid diffusion mode. Each cell gives the percentage impact of the parameter (row) on the indicator (column).

Road diffusion model. The Figures 13 display the 42 time series produced by the exploration of the roads diffusion model in the parameters decay rate and diffusion rate space. The observations are quite similar with the difference that the time series groups are much clearer: to confirm this observation, we conduct another ANOVA. A second observation is that AQI values are much higher than with the grid diffusion model.

Table 3 summarizes the ANOVA results. The analysis shows once again that the parameter with the most influence is Decay. But with the road diffusion model the impact of Diffusion and Decay-Diffusion parameters are definitely negligible (this appears clearly on the Figure 12 with clusters of series with the Decay value). No result can be computed for the minimum AQI indicator as results are constant (equal to 0.0). Finally, randomness appears to have even more impact in the road diffusion model than with the grid one.

\subsection{Effect of the number of vehicles}

The main objective of this experiment is to explore the impact of the number of vehicles. In particular, the key questions we want to address are: is there any qualitative change in the dynamics when the number of vehicles increase? How 


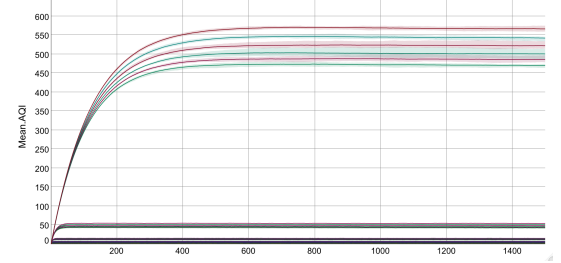

(a) Mean of AQI

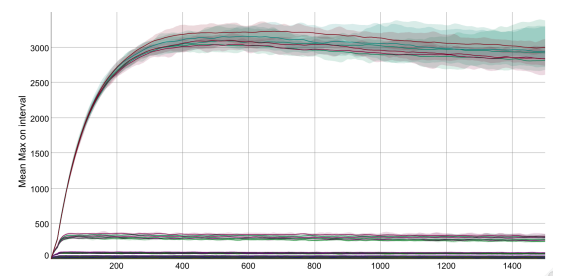

(c) Max of AQI

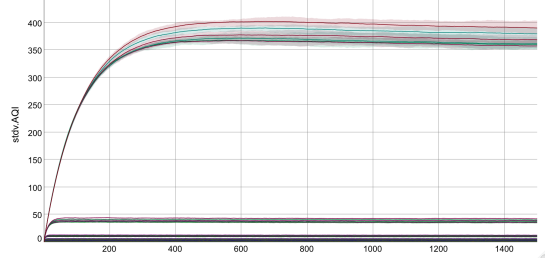

(b) Standard Deviation of AQI

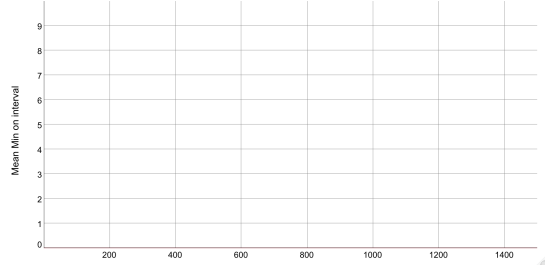

(d) Min of AQI

Fig. 13: Mean values of AQI indicators for the roads diffusion model with decay rate among $\{0.01,0.1,0.3,0.5,0.7,0.9,0.99\}$ and diffusion rate among $\{0.02,0.04,0.06,0.08,0.1,0.12\}$. Clusters of time series with the same value of decay illustrate the high sensibility of the model to this single parameter.

\begin{tabular}{|c|c|c|c|c|}
\hline & mean & stdDev & max & min \\
\hline \hline Decay & 38.61 & 39.46 & 39.23 & NaN \\
\hline Diffusion & 0.07 & 0.02 & 0.01 & NaN \\
\hline Decay:Diffusion & 0.16 & 0.05 & 0.02 & NaN \\
\hline Residuals & 61.16 & 60.48 & 60.74 & NaN \\
\hline
\end{tabular}

Table 3: Results of the ANOVA with the grid diffusion mode. Each cell gives the percentage impact of the parameter (row) on the indicator (column).

AQI indicators evolve when the number of vehicles increases and in particular is there a cap value?

To this purpose, we design an experiment with the parameter traffic factor taken values among $\{0.5,1.0,1.5,2.0,5.0\}$. This means that the number of vehicles varies between 350 cars and 1000 motorbikes (when traffic factor is equal to 0.5 ) to 3500 cars and 10000 motorbikes (when traffic factor $=5.0$ ). The ratio between cars and motorbikes is thus preserved in all the simulations. Results (on 10 replications) are plotted in Figures 14.

We observe a linear evolution of all the indicators with the multiplication factor traffic factor: as an example, when the mean AQI value at step=400 is around 40 (for traffic factor $=0.5$ ), it values 400 for traffic factor $=5.0$. In particular, results do not show a cap value of the AQI when the number of vehicle varies (as we observed when the step duration increases). Nevertheless, 


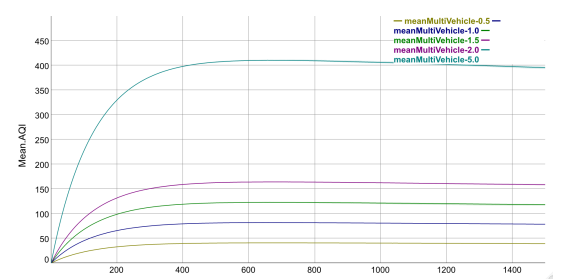

(a) Mean of AQI

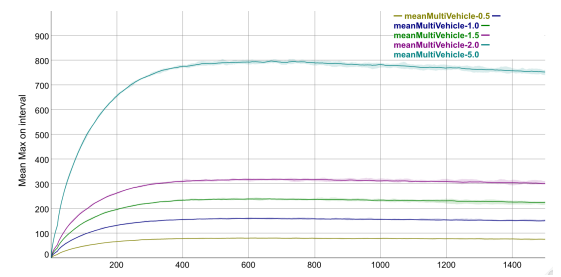

(c) Max of AQI

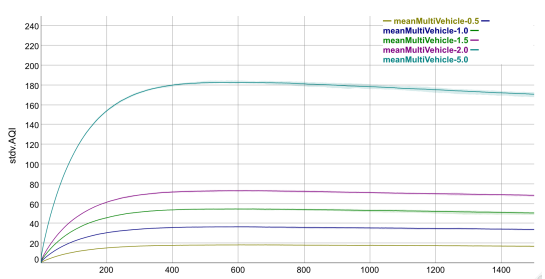

(b) Standard Deviation of AQI

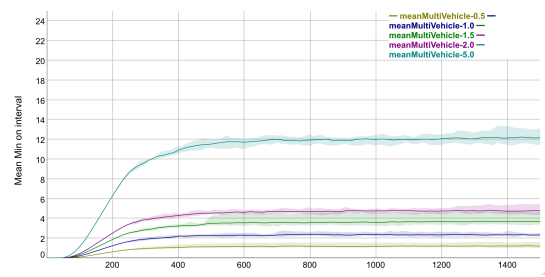

(d) Min of AQI

Fig. 14: Mean values of AQI indicators for traffic factor in $\{0.5,1.0,1.5,2.0,5.0\}$. Plots show a linear increase of the AQI indicators with the number of vehicles.

the shape of the evolution of all the indicator is similar for all the values of traffic factor.

\subsection{Effect of the traffic scenario}

The evolution of the number of vehicles in the area can follow two simulation modes: constant traffic or daytime traffic. In this experiment, we explore the impact of the daytime traffic scenario on the behavior of the simulations. The numbers of cars and motorbikes will thus change each hour, following data illustrated in Figure 3; but every day, the hourly numbers will be unchanged. We thus launched 10 replications for each value of the parameter traffic scenario (the simulations where the parameter value is set to constant traffic are used as a baseline). We launch simulations with a step of 5 minutes (1 day is thus simulated in 288 steps) and we stop the simulations after 3000 steps. Results are displayed in Figures 15.

As expected, the AQI values follows the number of vehicles: data (presented Figure 3) show 3 rush hours (around 7AM, 11AM and 5PM) and a low-traffic period during the night. The 3 local maximum and the minimum can clearly be observed on all the indicators (except the minimum of AQI). The extreme values intervals are very small showing the low impact of the stochasticity on the results when the number of vehicles evolves over time (the main exception is for the optimums of the max and min of AQI). The most interesting observation is the fact that indicator values are periodic (after the first day). The simulation bootstrap period is thus limited to the first day and after the second day, results are stabilized. 


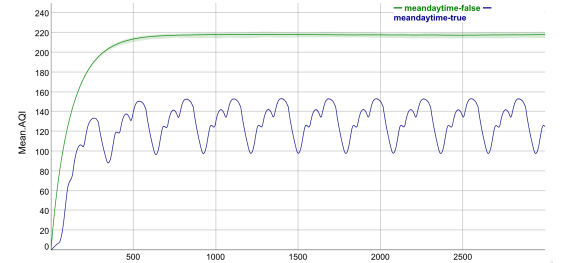

(a) Mean of AQI

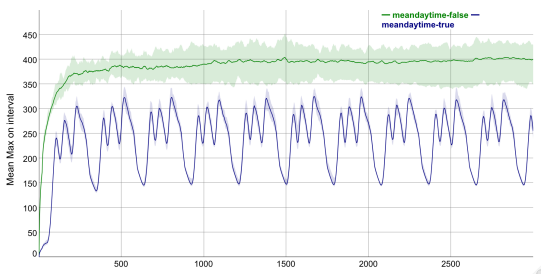

(c) Max of AQI

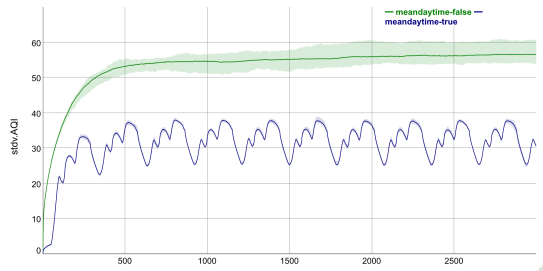

(b) Standard Deviation of AQI

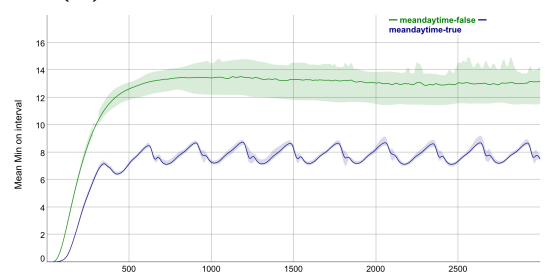

(d) Min of AQI

Fig. 15: AQI indicator results for each traffic scenario: green line for a number of vehicles constant traffic, purple for a number of vehicles following daytime traffic. Plots show that pollutant emissions become stationary after one day of simulation and correlated to the evolution of the number of vehicles.

\subsection{Effect of pedestrian area scenario}

The ultimate objective of this section is to explore the impact of the various pedestrian area scenarios on the AQI. We thus launched simulations with the pedestrian area scenario parameter taken values among \{no pedestrian area, current scenario, extended area\}, for both traffic scenarios (daytime or constant traffic). In addition, as the user is able to change the pedestrian area in the interactive mode, it appears necessary to check the impact of this change on indicators to prevent unstable states; this will also be conducted on both traffic scenarios.

Simulations of the pedestrian area scenario in constant traffic mode. In this experiment, we set the traffic mode to constant traffic, and thus set the simulation step duration to its default value (16s). We launched 10 replications for each pedestrian area scenario. Results are summarized in Figures 16.

A first notable observation is that the mean values of AQI are not significantly different among the three pedestrian scenarios. More generally, the 2 scenarios no pedestrian area and current scenario produce very similar results on all the indicators; the main difference is on the max AQI, which is higher for the current scenario. But as the extreme values intervals overlap, this difference is not really significant. The extended area scenario produces some differences in its results: the main one being a standard deviation value that is higher than for the 2 other scenarios and a slightly higher maximum of AQI. As a consequence, the extended area scenario is the one producing the most change, with higher maximum of 


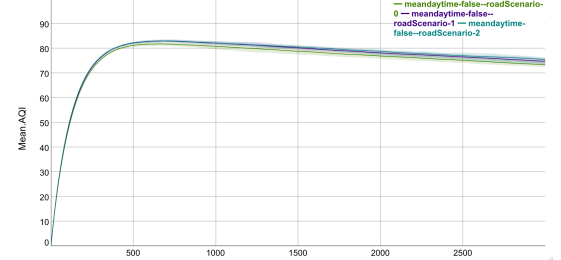

(a) Mean of AQI

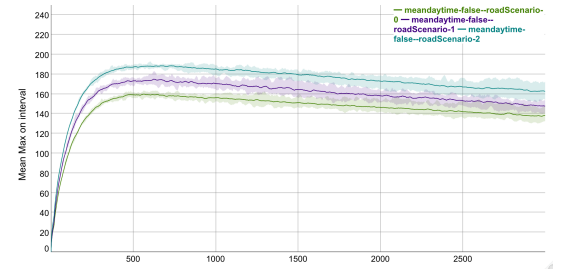

(c) Max of AQI

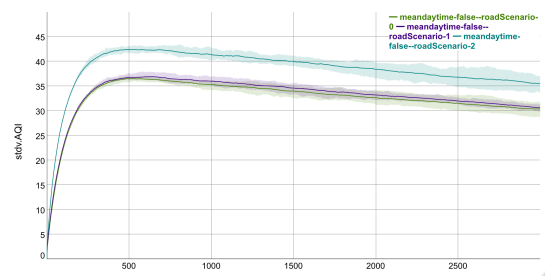

(b) Standard Deviation of AQI

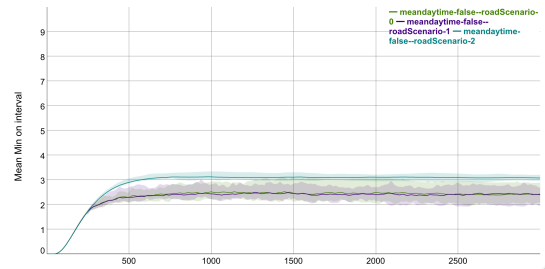

(d) Min of AQI

Fig. 16: Simulation results for the three scenarios of pedestrian area: green line for no pedestrian area, purple for current scenario, and indigo blue for extended scenario, with a constant number of vehicles. Figures show that the application of any of the pedestriation scenario does not change qualitatively the pollutant emissions, but only increase the maximum value and the spatial distribution heterogeneity.

AQI and a distribution of AQI among the buildings that is more heterogeneous than for the other scenarios.

Simulations of the pedestrian area scenario in daytime traffic mode. In this experiment, we set the traffic mode to daytime traffic, and thus the simulation step duration to $5 \mathrm{~min}$. We launched 10 replications for each pedestrian area scenario. Results are summarized in Figures 17.

Once again, we can observe that no pedestrian area and current scenario produce very similar results on all the indicators. The only slight difference is that during rush hours, the mean AQI is a little bit higher when pedestrian area is activated. Contrarily to the previous experiment, when the daytime traffic is activated, all the indicators increase when the extended scenario is activated: the AQI is higher in average, and so are the max and standard deviation. In this specific configuration, we can conclude that the extended pedestrian area increases not only the peak of AQI but also the overall pollutant quantities.

Effects of pedestrian area scenarios changes. During simulations, through the remote control, users can decide to change the pedestrian area scenario to observe the impacts of alternatives. To assess the impact of this switch between scenarios, we implement a new experiment in which the scenario is initialized to no pedestrian area. During the simulation, every 864 steps (in daytime mode, 


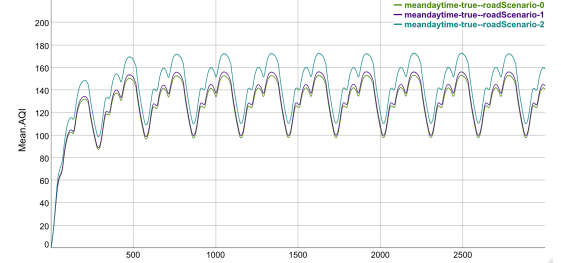

(a) Mean of AQI

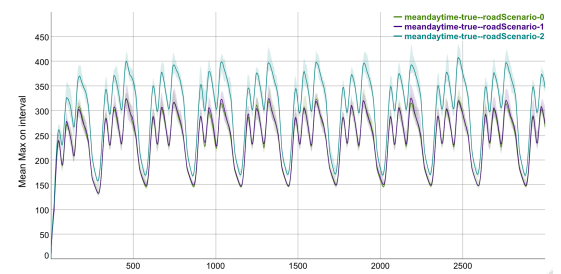

(c) Max of AQI

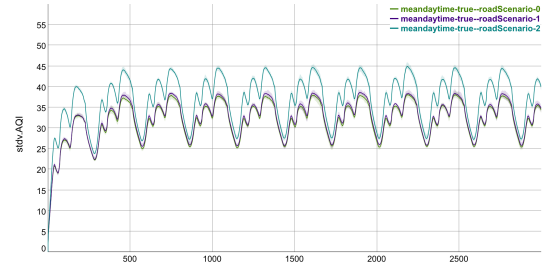

(b) Standard Deviation of AQI

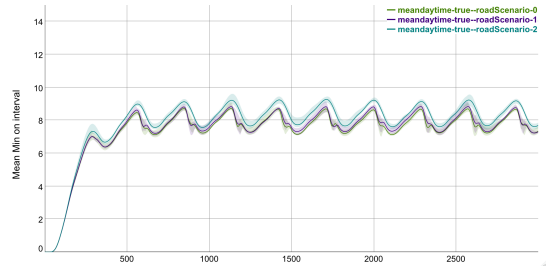

(d) Min of AQI

Fig. 17: Simulation results for the three scenarios of pedestrian area: green line for no pedestrian area, purple for current scenario, and indigo blue for extended scenario. In a situation of varying traffic, the application of each of the scenario from the beginning of the simulation highlight the fact that the situations without any pedestrian area and with the current scenario show both quantitatively and qualitatively similar results. In contrary, the extended scenario show an increase of the amount of the air pollutants and of their spatial distribution heterogeneity. 
i.e. 3 days) or 800 steps (in constant traffic mode), the scenario is changed: if the current scenario is no pedestrian area then it becomes current scenario (resp. extended area), otherwise it becomes no pedestrian area. As we run 3000 simulation steps, we will observe the alternation: no pedestrian area-current scenario-no pedestrian area-current scenario. Results are summarized in Figures 18 for the current traffic mode and Figures 19 for the daytime traffic mode.

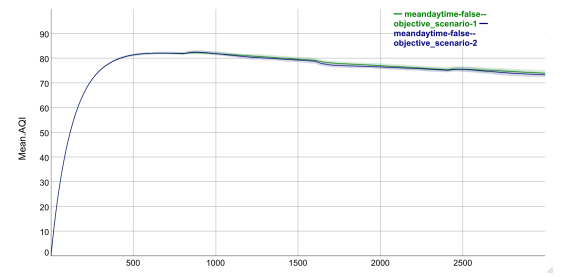

(a) Mean of AQI

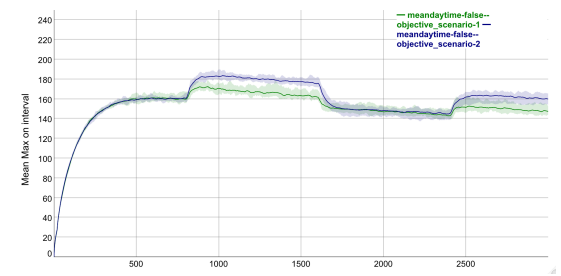

(c) Max of AQI

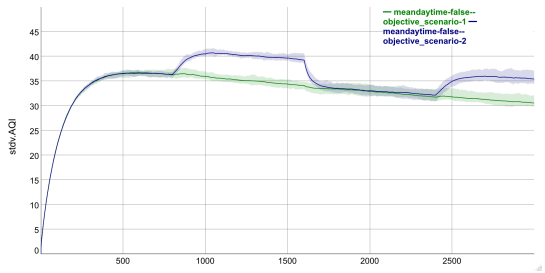

(b) Standard Deviation of AQI

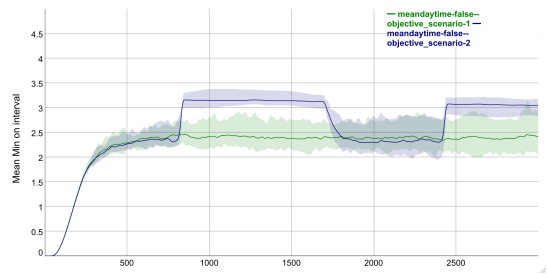

(d) Min of AQI

Fig. 18: Simulation (in constant traffic mode) results when the scenario of pedestrian area changes during the simulation (green line represents results with for current scenario and purple lines with extended scenario). In a hypothetical situation of constant traffic, these figures show that the application of the two alternative scenarios does not change the overall quantity of air pollutant but induces an increase of the maximum peak value. The application of the extended scenario is also characterized by a huge increase of the spatial distribution heterogeneity.

When the traffic mode is set to constant traffic, we observe, as previously, that the activation of any pedestrian scenario does not modify significantly the mean of AQI (even if a very small increase can be observed on the time series in Figure 18a). The change is more significant for the standard deviation (when activating the extended area scenario) and the max value of AQI (with a higher max value for the extended area scenario). Finally, we can observe that, when the pedestrian area scenario is switched from current or extended area scenario to no pedestrian area, the indicator values does go back to the baseline value immediately: on average it will take around 100 step to reach the step without scenario switch. 


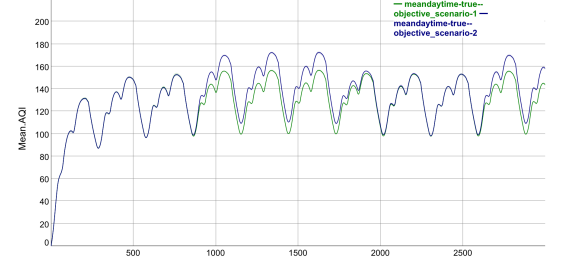

(a) Mean of AQI

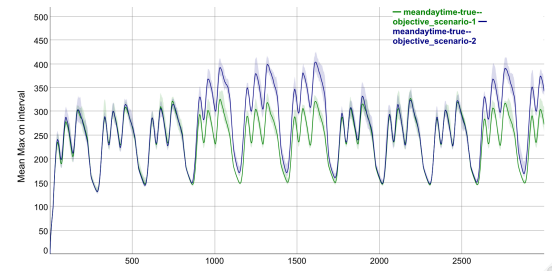

(c) Max of AQI

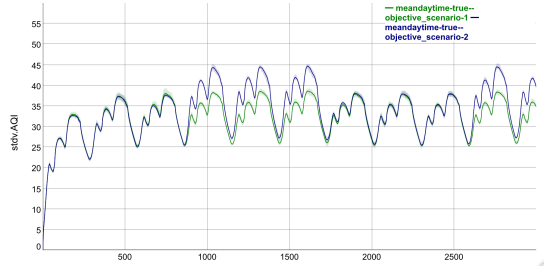

(b) Standard Deviation of AQI

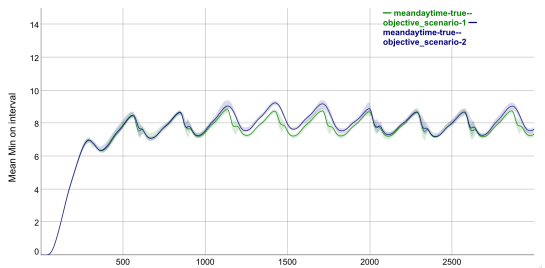

(d) Min of AQI

Fig. 19: Simulation (in daytime traffic mode) results when the scenario of pedestrian area changes during the simulation: green line represents results with for current scenario and green lines with extended scenario. In a situation of traffic varying over the day, these figures show that the extended scenario application induces an increase of the amount of air pollutants and of their spatial distribution heterogeneity. Conversely, the application of the current scenario does not show significant modification on these indicators. 
When the daytime traffic mode is activated, we observe similar results to the previous experiment concerning the impact of the pedestrian area scenario on the indicators. The mean AQI is not significantly impacted by the scenario as Figure 19a does not show clear difference between the period without pedestrian area (3 first days, i.e. before step 850) and period with a pedestrian area (next 3 days, roughly between steps 850 and 1700). Conversely, when the extended area scenario is activated, an increase in all the indicators is visible.

More interestingly, we can observe that, if we omit the initial bootstrap phase of the first day (that has been identified in the previous experiment), no transition phase appears later when switching between no pedestrian area and current scenario. In particular, it would have been possible to have an unstable period after each transition between pedestrian area scenarios, but the results of the first day of a period appears very similar to the other days of the period. Nevertheless, when the extended area scenario is deactivated, we can observe that the mean AQI values of the two series are slightly different during the first day after the switch, meaning that simulation requires one day to fully recovered from the pedestrian area scenario change (even if the difference between both series is not huge).

\section{Conclusion}

This article presents the HoanKiemAir project that aims at providing a tangible and interactive simulation to represent the impact of various pedestrian area scenarios around the Hoan Kiem lake (Hanoi, Vietnam), on traffic and emitted air pollutants. It is expected to help authorities and people audiences to understand the interrelations and feedback loops between these dynamics using a 3D map as a screen for the simulation and a tablet application to control it. Whereas the model is described in details in [28], this paper focuses on the analysis of the simulator and in particular on the sensibility of the results when exploring the parameter space.

The results of experiments presented here tend to show that the step duration can be changed without changing qualitatively the results, and that there is a threshold above which, it does not have any quantitative impact either. Conversely, not such a threshold can be identified on the number of vehicles: the increase of the number of vehicles induces a linear increase on the AQI indicators. As far as the traffic scenario is concerned, the evolution of AQI is coherent with the traffic evolution in the daytime traffic and a bootstrap period of 1 day as been identified. Finally, the activation of the current pedestrian area does not significantly impact the AQI (even if we can notice a small increase of the maximum of AQI) in both constant and daytime traffic configurations. Concerning the extended area scenario, its activation definitely increases the maximum of AQI and the standard deviation (i.e. increases the heterogeneity of the spatial AQI distribution). In the case of the daytime traffic, it also increases the mean AQI (and thus the overall quantity of pollutants in the system). When a switch is made between the extended area and the no pedestrian area, we can observe 
that a period of time is necessary to come back to state before the activation of the pedestrian area (around 100 steps in constant traffic and 1 day for daytime traffic).

The analysis of the simulation behaviour when exploring the parameter values reinforce the trust we can have on the use of the simulator in an interactive way with various audience. Obviously the model still need to be improved both with additional data about the real traffic in the area and the other pollution sources (factory, agriculture, etc), and additional dynamics, and in particular a weather model and how it interacts with the pollution diffusion model. Finally, we still need to calibrate the model more accurately with real data, mainly coming from sensors localized in the area.

In the end, apart from this theoretical analysis of the model, the full setup has been demonstrated several times to diverse audiences. Preliminary observations show that the use of a 3D printed map definitely attracts people and arouses their interest and curiosity. Feedback were very positive. Nevertheless, we have observed that the full setup in its current state is not fully self-content: when users are alone (i.e. without any facilitator around) in front of the map and simulation, they are not able, in general, to fully understand what they observe. There is still a need to improve contextual information (either on the simulation or around the table). A second point is that the tablet application is not naturally used by the users. An interesting alternative could be to build a physical remote control with actual sliders and buttons to evaluate if users are more likely to use it rather than the Android application.

The simulation (in an extended version) could be used by both decisionmakers and urban planners to test several scenarios of pedestrian areas and compare their results in an interactive way. Extensions of the interactions with the model could allow the user to define interactively the pedestrian area to test, at the scale of the single road. This would give insights about the amount of roads that can be closed before the appearance of critical consequences in the other areas of the district and even in other districts. This could also allow decision-makers to identify key roads, roads which will highly and even qualitatively impact the overall traffic and air pollution emission. As far as the public is concerned, the simulation results would help it to understand the complex feedback loop between the urban-planning decisions and traffic and air pollutants emission. This could help it to accept a debatable or provide it elements to factually criticize it. We could also imagine using the setup to let people propose new pedestrian plans in a crowd sourcing approach.

\section{Acknowledgement}

The HoanKiemAir project was funded by the French embassy in Vietnam, in collaboration with the Hoan Kiem district People's Committee and PRX Vietnam. 


\section{References}

1. ALMEC Corp, Nippon Koei Co Ltd, Yachiyo Engineering Co Ltd: The comprehensive urban development programme in Hanoi Capital City of the Socialist Republic of Vietnam (HAIDEP) (2007)

2. Alonso, L., Zhang, Y.R., Grignard, A., Noyman, A., Sakai, Y., ElKatsha, M., Doorley, R., Larson, K.: Cityscope: a data-driven interactive simulation tool for urban design. use case volpe. In: International Conference on Complex Systems. pp. 253-261. Springer (2018)

3. Arnaud, B.: Simulating pedestrian behavior in complex and dynamic environments: An agent-based perspective. In: F., B., Mager, C. (eds.) European Handbook of Theoretical and Quantitative Geography, pp. 1-27. Faculty of Geosciences and Environment of the University of Lausanne (2009)

4. Banos, A., Corson, N., Lang, C., Marilleau, N., Taillandier, P.: Multiscale modeling: Application to traffic flow. In: Agent-based Spatial Simulation with NetLogo, Volume 2, pp. 37-62. Elsevier (2017)

5. Bigazzi, A.Y., Rouleau, M.: Can traffic management strategies improve urban air quality? a review of the evidence. Journal of Transport \& Health 7, 111-124 (2017)

6. Chao, Q., Bi, H., Li, W., Mao, T., Wang, Z., Lin, M.C., Deng, Z.: A survey on visual traffic simulation: Models, evaluations, and applications in autonomous driving. Computer Graphics Forum 39(1), 287-308 (2020)

7. Chapuis, K., Taillandier, P., Gaudou, B., Drogoul, A., Daudé, E.: A multi-modal urban traffic agent-based framework to study individual response to catastrophic events. In: International Conference on Principles and Practice of Multi-Agent Systems. pp. 440-448. Springer (2018)

8. Chifflet, S., Amouroux, D., Bérail, S., Barre, J., Van, T.C., Baltrons, O., Brune, J., Dufour, A., Guinot, B., Mari, X.: Origins and discrimination between local and regional atmospheric pollution in haiphong (vietnam), based on metal (loid) concentrations and lead isotopic ratios in pm 10. Environmental Science and Pollution Research 25(26), 26653-26668 (2018)

9. Cohen, P.R.: Empirical methods for artificial intelligence, vol. 139. MIT press Cambridge, MA (1995)

10. Emery, J., Marilleau, N., Martiny, N., Thévenin, T., Nguyen-Huu, T., Badram, M., Grignard, A., Hbdid, H., Laatabi, A.M., Toubhi, S.: Marrakair: une simulation participative pour observer les émissions atmosphériques du trafic routier en milieu urbain. In: Treizièmes Rencontres de Théo Quant (2017)

11. Fleck, S., Rivière, G., Ticona-Herrera, R., Couture, N.: Classifying tangible user interfaces with cladistics: criteria and forms for a collaborative inventory. In: Proceedings of the 30th Conference on l'Interaction Homme-Machine. pp. 209-218 (2018)

12. Grignard, A., Alonso, L., Taillandier, P., Gaudou, B., Nguyen-Huu, T., Gruel, W., Larson, K.: The impact of new mobility modes on a city: A generic approach using abm. In: International Conference on Complex Systems. pp. 272-280. Springer (2018)

13. Grignard, A., Macià, N., Alonso Pastor, L., Noyman, A., Zhang, Y., Larson, K.: Cityscope Andorra: a multi-level interactive and tangible agent-based visualization. In: Proc. of AAMAS. pp. 1939-1940 (2018)

14. Grimm, V., Berger, U., Deangelis, D., Polhill, J., Giske, J., F. Railsback, S.: The ODD protocol: A review and first update. Ecological Modelling 221, 2760-2768 (2010) 
15. Hertel, O., Berkowicz, R.: Operational Street Pollution Model (OSPM). evaluation of the model on data from St. Olavs street in Oslo. Tech. rep., National Environmental Research Institute, Danish Ministry of the Environment (01 1989)

16. Hoogendoorn, S.P., Bovy, P.H.L.: State-of-the-art of vehicular traffic flow modelling. Journal of Systems and Control Engineering 215(4), 283-303 (2001)

17. Kobayashi, K., Narita, A., Hirano, M., Kase, I., Tsuchida, S., Omi, T., Kakizaki, T., Hosokawa, T.: Collaborative simulation interface for planning disaster measures. In: CHI'06 Extended Abstracts on Human Factors in Computing Systems. pp. 977-982 (2006)

18. Kubicki, S., Lepreux, S., Kolski, C.: Rfid-driven situation awareness on tangisense, a table interacting with tangible objects. Personal and Ubiquitous Computing 16(8), 1079-1094 (2012)

19. Laatabi, A., Becu, N., Marilleau, N., Pignon-Mussaud, C., Amalric, M., Bertin, X., Anselme, B., Beck, E.: Mapping and describing geospatial data to generalize complex models: The case of LittoSIM-GEN. International Journal of Geospatial and Environmental Research 7(1), 6 (2020)

20. Lebrun, Y., Adam, E., Mandiau, R., Kolski, C.: A model for managing interactions between tangible and virtual agents on an RFID interactive tabletop: case study in traffic simulation. Journal of Computer and System Sciences 81(3), 585-598 (2015)

21. Lighthill, M.J., Whitham, G.B.: On kinematic waves II. A theory of traffic flow on long crowded roads. Proceedings of the Royal Society of London. Series A. Mathematical and Physical Sciences 229(1178), 317-345 (1955)

22. Macal, C., North, M.: Tutorial on agent-based modelling and simulation. J. Simulation 4, 151-162 (2010)

23. Mitchell, M.: Complexity : a guided tour. Oxford University Press (2009)

24. Nagel, K., Schreckenberg, M.: A cellular automaton model for freeway traffic. Journal de physique I 2(12), 2221-2229 (1992)

25. Ntziachristos, L., Gkatzoflias, D., Kouridis, C., Samaras, Z.: COPERT: A European road transport emission inventory model. Information Technologies in Environmental Engineering pp. 491-504 (01 2009)

26. Organization, W.H.: WHO releases country estimates on air pollution exposure and health impact (2016), https://www.who.int/news-room/detail/ 27-09-2016-who-releases-country-estimates-on-air-pollution-exposure-and-health-impact

27. Petrasova, A., Harmon, B., Petras, V., Mitasova, H.: Tangible modeling with open source GIS. Springer (2015)

28. Pham, M.D., Chapuis, K., Drogoul, A., Gaudou, B., Grignard, A., Marilleau, N., Tri, N.H.: HoanKiemAir: simulating impacts of urban management practices on traffic and air pollution using a tangible agent-based model. In: The 2020 RIVF International Conference on Computing and Communication Technologies. pp. 17. IEEE (2020)

29. Richards, P.I.: Shock waves on the highway. Operations research 4(1), 42-51 (1956)

30. Rodrique, K., Ho, T., Nguyen, M.H.: An agent-based simulation for studying air pollution from traffic in urban areas: The case of Hanoi city. IJACSA 10, 596-604 (04 2019)

31. Salim, F.: Tangible 3D urban simulation table. In: Proceedings of the Symposium on Simulation for Architecture \& Urban Design. vol. 23. Society for Computer Simulation International (2014)

32. Saltelli, A., Chan, K., Scott, E.M.: Sensitivity analysis (Vol. 1). Wiley, New York (2000) 
33. Taillandier, P., Gaudou, B., Grignard, A., Huynh, Q.N., Marilleau, N., Caillou, P., Philippon, D., Drogoul, A.: Building, composing and experimenting complex spatial models with the GAMA platform. GeoInformatica 23(2), 299-322 (2019)

34. Tho Hung, N., Ketzel, M., Jensen, S., Oanh, N.T.: Air pollution modeling at road sides using the operational street pollution model-a case study in hanoi, vietnam. Journal of the Air \& Waste Management Association (1995) 60, 1315-26 (11 2010)

35. Underwood, R.T.: Speed, volume and density relationships. Quality and theory of traffic flow (1961)

36. Vietnam Env. Admin.: Quyet dinh 878/QD-TCMT ve viec ban hanh so tay huong dan tinh toan chi so chat luong khong khi (AQI) (2011)

37. van Wageningen-Kessels, F., Van Lint, H., Vuik, K., Hoogendoorn, S.: Genealogy of traffic flow models. EURO Journal on Transportation and Logistics 4(4), 445-473 (2015)

38. Woods, T.L., Reed, S., Hsi, S., Woods, J.A., Woods, M.R.: Pilot study using the augmented reality sandbox to teach topographic maps and surficial processes in introductory geology labs. Journal of Geoscience Education 64(3), 199-214 (2016)

39. Zhang, K., Batterman, S.: Air pollution and health risks due to vehicle traffic. Science of the total Environment 450, 307-316 (2013) 